\title{
CORRELAÇÃO DO ESQUEMA DE IMUNOSSUPRESSÃO COM COMPLICAÇÕES PÓS-OPERATÓRIAS EM TRANSPLANTES RENAIS ATRAVÉS DO USO DA CINTILOGRAFIA RENAL DINÂMICA*
}

\author{
Flávia Paiva Proença Martins', Renato Teixeira Gonçalves², Léa Mirian Barbosa da Fonseca ${ }^{3}$, \\ Bianca Gutfilen ${ }^{4}$
}

Resumo A cintilografia renal dinâmica possibilita o diagnóstico de complicações obsenvadas nos tecidos transplantados, como desordens na perfusão do órgão, necrose tubular aguda e quadros de rejeição. Empregamos o פmTc-DTPA neste estudo e correlacionamos os achados cintilográficos e clínicos visando ao diagnóstico de rejeição ou outra forma de complicação no órgão transplantado. Tanto as rejeições quanto as complicações foram avaliadas em relação ao tipo de imunossupressão utilizada. Foram analisados 55 pacientes submetidos a transplante renal entre 1989 e 1999. Todos os pacientes com nefrotoxicidade faziam uso do esquema tríplice de imunossupressão. Neste estudo houve predominância de rejeição aguda, em $40,4 \%$ dos casos. Treze dos quinze pacientes cujos doadores eram cadáveres tiveram necrose tubular aguda. Foi observado apenas um caso falso-positivo, em que o exame cintilográfico foi incompatível com a clínica. Sugerimos o uso da cintilografia renal no acompanhamento pós-operatório dos pacientes transplantados.

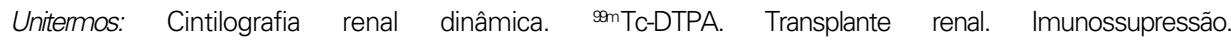

Abstract Correlation of immunosuppression scheme with renal graft complications detected by dynamic renal scintigraphy.

Dynamic renal scintigraphy allows the diagnosis of complications in patients submitted to organ transplantation, such as perfusion abnormalities, acute tubular necrosis and rejection. In this study we employed ${ }^{\text {mm} T c-~}$ DTPA scintigraphy to study patients submitted to kidney transplantation. The results obtained and the clinical findings were conjunctly analyzed in order to detect graft rejection or other complications. The type of immunosuppressive scheme used was also correlated with the observed complications. Fifty-five patients submitted to kidney transplantation from 1989 to 1999 were evaluated. All patients with nephrotoxicity received a 3-drug immunosuppressive scheme. In this study, acute rejection was the most frequent complication (40.4\%) observed following transplantation. Thirteen of 15 recipients of cadaveric kidney grafts presented acute tubular necrosis. Only one false-positive case was observed when scintigraphy and clinical findings were not concordant. We suggest carrying out renal scintigraphy to follow-up post-transplantation patients. Key words: Dynamic renal scintigraphy. somTc-DTPA. Kidney transplantation. Immunosuppression.

\section{INTRODUÇÃO}

Atualmente, a substituição de um órgão irremediavelmente doente é prática clínica diária; entretanto, o conhecimento imunológico ainda é incompleto e muitos órgãos são perdidos por causa de rejeição ${ }^{(1)}$.

* Trabalho realizado no Departamento de Radiologia da Universidade Federal do Rio de Janeiro (UFRJ), Rio de Janeiro, RJ.

1. Biomédica da Universidade do Rio de Janeiro (Unirio), Estagiária do Departamento de Radiologia da UFRJ.

2. Médico do Senviço de Nefrologia, Coordenador da Unidade de Transplante Renal do Hospital Universitário Clementino Fraga Filho (HUCFF) da UFRJ.

3. Professora Titular de Medicina Nuclear da UFRJ.

4. Biomédica da Universidade Estadual do Rio de Janeiro (UERJ), Doutora em Medicina Nuclear da UFRJ.

Endereço para correspondência: Dra. Bianca Gutfilen. Universidade Federal do Rio de Janeiro, Hospital Universitário Clementino Fraga Filho. Avenida Brigadeiro Trompovsky, $s / n^{\circ}$. Rio de Janeiro, RJ, 21941-590. E-mail: audio company@unikey.com.br

Aceito para publicação em 8/6/2001.
De acordo com a clínica e o período de aparecimento, as rejeições são classificadas como hiperagudas, agudas e crônicas. Essas rejeições levam a um declínio da função renal e necessitam de intervenção rápida para se evitar a perda do enxerto ${ }^{(1)}$.

Existem dois tipos principais de rejeição: a aguda ou vascular, e a celular. Em boa parte dos casos, a rejeição aguda é grave, de ocorrência precoce e evolui para perda do enxerto. Em contraste, a celular é normalmente comum e se manifesta nas primeiras semanas ou meses ${ }^{(2)}$.

Contudo, existem métodos utilizados para minimizar a rejeição pós-operatória, como o uso de algumas drogas e radiação. Essas drogas são chamadas imunossupressores. Dentre estas, são comumente utilizadas a azatioprina, a prednisolona e a ciclosporina. Mais recentemente, vem sendo observado o uso do OKT3, um anticorpo monoclonal anti-CD3, que tem sido associado à reversão efetiva da rejeição de transplantes renais ${ }^{(3-6)}$.

Apesar dos benefícios, existem inúmeros efeitos colaterais causados pelos imunossupressores, como, por exemplo, a nefrotoxicidade ${ }^{(7,8)}$.

Sabemos que a utilização da medicina nuclear na avaliação do trato genitourinário confunde-se com as primeiras aplicações de radionuclídios. Assim, vem demonstrando ser um excelente método para o diagnóstico de rejeição renal. $\mathrm{O}$ potencial dos métodos nucleares para a detecção de alterações clínicas na estrutura anatômica, fisiológica e bioquímica é praticamente ilimitado ${ }^{(7,9-11)}$.

A maioria dos laboratórios de medicina nuclear utiliza o ácido dietilenotriaminopentacético marcado com tecné- 
cio-99m ( ${ }^{99 \mathrm{~m}}$ Tc-DTPA), adquirindo imagens do enxerto, durante 30 minutos. Sua utilização não é aleatória, visto que este composto é quase integralmente filtrado pelos rins (98\% de filtração), sem nenhuma reabsorção ou secreção tubular, oferecendo, assim, informações relevantes na avaliação da função glomerular ${ }^{(9)}$.

\section{CASUÍSTICA, MATERIAL E MÉTODOS}

Foram avaliadas 142 cintilografias renais de 68 pacientes pós-transplantados, realizadas no Serviço de Medicina $\mathrm{Nu}$ clear do Hospital Universitário Clementino Fraga Filho da Universidade Federal do Rio de Janeiro, no período compreendido entre maio de 1989 e dezembro de 1999. Os exames foram realizados entre 24 e 48 horas após a cirurgia e, posteriormente, nos casos em que houve suspeita clínica de rejeição ao enxerto ou necrose tubular aguda.

Foram considerados os achados clínicos obtidos da requisição do exame cintilográfico ou das informações obtidas da análise dos prontuários. Os seguintes parâmetros foram utilizados para o diagnóstico clínico: a) disfunção do enxerto renal, caracterizada por elevados índices plasmáticos de creatinina (> 2,0 mg/dl) e função retardada do enxerto; b) febre; c) desconforto na região do transplante; d) oligúria; e) variações da pressão arterial.

Os pacientes que não retornaram ao hospital para o acompanhamento clínico pós-cirúrgico foram excluídos. As cintilo- grafias selecionadas mostravam casos de rejeição aguda (RA), nefropatias crônicas (NC), processos de necrose tubular aguda (NTA), trombose, ou, ainda, aspectos considerados normais para um rim transplantado. O grupo de estudo final foi composto de 55 pacientes, perfazendo um total de 127 exames.

A confirmação dos casos foi obtida pela evolução clínica e/ou achados histopatológicos.

Os exames foram realizados em dois tipos de gama câmara: a câmara de cintilação planar tipo Anger, marca Ohio-Nuclear, Sigma 410, acoplada a computador Alfa Nuclear S.A.I. yC, e a gama câmara Diacan, acoplada a computador Siemens. A descrição de dois tipos de câmaras cintilográficas foi feita por se tratar de estudo retrospectivo; assim sendo, a câmara foi substituída no decorrer dos anos.

Foi utilizado um protocolo de estudo para a análise dos pacientes, em que foram ressaltados os achados cintilográficos correlacionados com a clínica.

Alguns parâmetros relacionados à imunossupressão utilizada pelos receptores e às complicações ocorridas após a cirurgia foram analisados. Os diferentes tipos de identidades imunológicas também foram considerados.

Os pacientes foram divididos em grupos, de acordo com o tipo de imunossupressão administrada: grupo I - esquema tríplice; grupo II - esquema duplo.

No esquema tríplice de imunossupressão são administrados, concomitantemente, $2 \mathrm{mg} / \mathrm{kg}$ de azatioprina, $0,5 \mathrm{mg} /$ $\mathrm{kg}$ de prednisolona e $8 \mathrm{mg} / \mathrm{kg}$ de ciclosporina, e no esquema duplo de imunossupressão são administrados $3 \mathrm{mg} / \mathrm{kg}$ de azatioprina e $1 \mathrm{mg} / \mathrm{kg}$ de prednisolona.

\section{RESULTADOS}

Na Tabela 1 são mostrados a relação dos casos estudados com as respectivas identidades imunológicas, a imunossupressão utilizada, as complicações pósoperatórias e os resultados cintilográficos do transplante.

As complicações pós-operatórias foram divididas em NTA, NC, RA, trombose, nefrotoxicidade e síndrome hemolítica urêmica (SHU). Em 16 casos foi diagnosticada NTA, confirmada pela cintilografia renal e, posteriormente, pela histopatologia. Destes pacientes, 87,5\% (14 pacientes) eram receptores de enxertos provenientes de cadáveres. Foram também observados sete casos de NC, além de 19 casos de RA e um caso de trombose. Um paciente com SHU e cinco com nefrotoxicidade foram detectados, sendo que todos eles faziam uso do esquema tríplice de imunossupressão.

Os padrões normais da cintilografia renal com ${ }^{99 m}$ Tc-DTPA, após o transplante, são mostrados na Figura 1 (caso 6). Na Figura 2 (caso 52) é mostrado um caso de NTA diagnosticado por cintilografia re nal feita com ${ }^{99 m}$ Tc-DTPA. Um caso de rejeição é mostrado na Figura 3 (caso 36).

Dos 55 pacientes estudados, 36 tiveram boa aceitação ao enxerto. Foram incluídos, nesta classificação, não apenas

Tabela 1 Relação do esquema de imunossupressão utilizado, identidade imunológica, complicações pós-operatórias apresentadas e resultados cintilográficos dos transplantes.

\begin{tabular}{|c|c|c|c|l|}
\hline Paciente & Imunossupressão & Identidade imunológica & Complicações & Resultados cintilográficos \\
\hline 1 & Esquema tríplice & "Match" pobre & NTA & Padrão de NTA \\
2 & Esquema tríplice & "Match" pobre & NTA & Padrão de NTA \\
3 & Esquema tríplice & "Match" pobre & NTA de NTA \\
4 & Esquema duplo & "Match" pobre & Sem complicação & Padrão normal \\
5 & Esquema tríplice & "Match" pobre & Sem complicação & Padrão normal \\
6 & Esquema tríplice & Idêntica & Sem complicação & Padrão normal \\
7 & Esquema tríplice & "Match" pobre & Sem complicação & Padrão normal \\
8 & Esquema tríplice & Idêntica & RA & Padrão de RA \\
9 & Esquema tríplice & Idêntica & RA/NC & Padrão de RA/padrão de NC \\
10 & Esquema duplo & Idêntica & Sem complicação & Padrão normal \\
\hline
\end{tabular}

(continua na página seguinte) 
Martins FPP et al.

Tabela 1 Relação do esquema de imunossupressão utilizado, identidade imunológica, complicações pós-operatórias apresentadas e resultados cintilográficos dos transplantes. (continuação)

\begin{tabular}{|c|c|c|c|c|}
\hline Paciente & Imunossupressão & Identidade imunológica & Complicações & Resultados cintilográficos \\
\hline 11 & Esquema duplo & Idêntica & Sem complicação & Padrão normal \\
\hline 12 & Esquema tríplice & Haplo & RA & Padrão de RA \\
\hline 13 & Esquema tríplice & Haplo & NTA & Padrão de NTA \\
\hline 14 & Esquema tríplice & Idêntica & Sem complicação & Padrão normal \\
\hline 15 & Esquema tríplice & Haplo & Sem complicação & Padrão normal \\
\hline 16 & Esquema tríplice & Haplo & Sem complicação & Padrão normal \\
\hline 17 & Esquema tríplice & Haplo & Sem complicação & Padrão normal \\
\hline 18 & Esquema tríplice & Haplo & $\begin{array}{l}\text { RA/nefrotoxicidade } \\
\text { à ciclosporina }\end{array}$ & Padrão de RA \\
\hline 19 & Esquema tríplice & Haplo & Sem complicação & Padrão normal \\
\hline 20 & Esquema tríplice & Haplo & Sem complicação & Padrão normal \\
\hline 21 & Esquema tríplice & Haplo & Sem complicação & Padrão normal \\
\hline 22 & Esquema tríplice & Haplo & Sem complicação & Padrão normal \\
\hline 23 & Esquema tríplice & Haplo & Sem complicação & Padrão normal \\
\hline 24 & Esquema tríplice & Haplo & $\begin{array}{l}\text { Nefrotoxicidade } \\
\text { à ciclosporina }\end{array}$ & Padrão de RA \\
\hline 25 & Esquema tríplice & Haplo & NTA/RA & Padrão de NTA/padrão de RA \\
\hline 26 & Esquema tríplice & Haplo & NTA & Padrão de NTA \\
\hline 27 & Esquema duplo & Haplo & RA & Padrão de RA \\
\hline 28 & Esquema tríplice & "Match" pobre & RA & Padrão de RA \\
\hline 29 & Esquema tríplice & "Match" pobre & Sem complicação & Padrão normal \\
\hline 30 & Esquema tríplice & "Match" pobre & $\mathrm{RA} / \mathrm{NC}$ & Padrão de RA/padrão de NC \\
\hline 31 & Esquema tríplice & "Match" pobre & RA & Padrão de RA \\
\hline 32 & Esquema tríplice & "Match" pobre & $\mathrm{RA} / \mathrm{NC}$ & Padrão de RA/padrão de NC \\
\hline 33 & Esquema tríplice & Idêntica & RA/nefrotoxicidade & Padrão de RA \\
\hline 34 & Esquema tríplice & Haplo & NC & Padrão de NC \\
\hline 35 & Esquema tríplice & Haplo & $\begin{array}{l}\text { RA/nefrotoxicidade } \\
\text { à ciclosporina }\end{array}$ & Padrão de RA \\
\hline 36 & Esquema tríplice & Haplo & RA & Padrão de RA \\
\hline 37 & Esquema tríplice & Haplo & RA & Padrão de RA \\
\hline 38 & Esquema tríplice & Haplo & $\mathrm{RA} / \mathrm{SHU}$ & Padrão de RA \\
\hline 39 & Esquema tríplice & "Match" pobre & NTA & Padrão de NTA \\
\hline 40 & Esquema tríplice & "Match" pobre & Sem complicação & Padrão normal \\
\hline 41 & Esquema tríplice & "Match" pobre & $\mathrm{RA} / \mathrm{NC}$ & Padrão de RA/padrão de NC \\
\hline 42 & Esquema tríplice & Haplo & Trombose & Padrão de RA \\
\hline 43 & Esquema tríplice & Haplo & $\mathrm{RA} / \mathrm{NC}$ & Padrão de RA/padrão de NC \\
\hline 44 & Esquema tríplice & Haplo & RA & Padrão de RA \\
\hline 45 & Esquema tríplice & "Match" pobre & Sem complicação & Padrão de RA \\
\hline 46 & Esquema tríplice & "Match" pobre & NTA & Padrão de NTA \\
\hline 47 & Esquema tríplice & "Match" pobre & NTA & Padrão de NTA \\
\hline 48 & Esquema tríplice & "Match" pobre & NTA & Padrão de NTA \\
\hline 49 & Esquema tríplice & "Match" pobre & NTA/nefrotoxicidade/NC & Padrão de NTA/padrão de NC \\
\hline 50 & Esquema tríplice & "Match" pobre & NTA & Padrão de NTA \\
\hline 51 & Esquema tríplice & "Match" pobre & NTA & Padrão de NTA \\
\hline 52 & Esquema tríplice & "Match" pobre & NTA & Padrão de NTA \\
\hline 53 & Esquema tríplice & "Match" pobre & NTA & Padrão de NTA \\
\hline 54 & Esquema tríplice & "Match" pobre & NTA & Padrão de NTA \\
\hline 55 & Esquema tríplice & Haplo & RA & Padrão de RA \\
\hline
\end{tabular}

NTA, necrose tubular aguda; RA, rejeição aguda; NC, nefropatia crônica; SHU, síndrome hemolítica urêmica. 
aqueles que não tiveram complicações no pós-operatório, como também os pacientes que se recuperaram das complicações. Na Tabela 2 estão relacionadas as identidades imunológicas dos pacientes, assim como a evolução dos transplantes. Observamos que os pacientes antígenos leucocitários humanos ("human leukocyte antigen" - HLA) idênticos tiveram as melhores evoluções (85,7\% dos casos).

O grupo I (esquema tríplice) consistiu de 51 pacientes. Destes, 16 tiveram RA; três, NC; 12, NTA; um, trombose; e um, SHU. Em nosso estudo, apenas os pacientes que utilizaram o esquema tríplice de imunossupressão desenvolveram nefrotoxicidade (cinco pacientes). Destes, em três casos a nefrotoxicidade foi conseqüente à ciclosporina (60\% dos casos). Com a utilização deste esquema de imunossupressão, observamos a aceitação do enxerto em 35 pacientes.

O grupo II (esquema duplo) foi formado por quatro pacientes, em que apenas um caso de RA foi observado. Os demais pacientes não tiveram complicações pósoperatórias, não sendo necessária a administração do esquema tríplice de imunossupressão. Embora um dos pacientes tenha cursado com quadro de RA, este foi revertido com a utilização apenas do esquema duplo de imunossupressão.

O estudo cintilográfico apresentou sensibilidade de $100 \%$, especificidade de $94,4 \%$, precisão de $98,2 \%$, valor preditivo positivo de $97,4 \%$ e valor preditivo negativo de $100 \%$.

\section{DISCUSSÃO E CONCLUSÃO}

Verificamos 18 dos 55 pacientes estudados sem complicações no pós-operatório. É descrito, na literatura, que cerca de $25 \%$ dos pacientes transplantados geralmente não desenvolvem complicações no enxerto. Mesmo na presença de um novo órgão, os receptores poderão ter aceitação total do enxerto sem disfunções renais, principalmente naqueles com fatores predisponentes para hipertensão, diabete e doenças arteriais coro$\operatorname{narianas}^{(7)}$.

Os três primeiros meses após o transplante são os mais críticos no que se refere ao aparecimento de sinais locais e

Tabela 2 Evolução dos transplantes $\times$ identidade imunológica.

\begin{tabular}{|l|c|c|}
\hline \multirow{2}{*}{ Identidade imunológica } & Evolução dos transplantes \\
\cline { 2 - 3 } & Boa & Má \\
\hline HLA idêntico & 6 & 6 \\
HLA haplo idêntico & 18 & 10 \\
HLA "match" pobre & 14 & 1 \\
\hline
\end{tabular}

HLA, antígeno leucocitário humano.

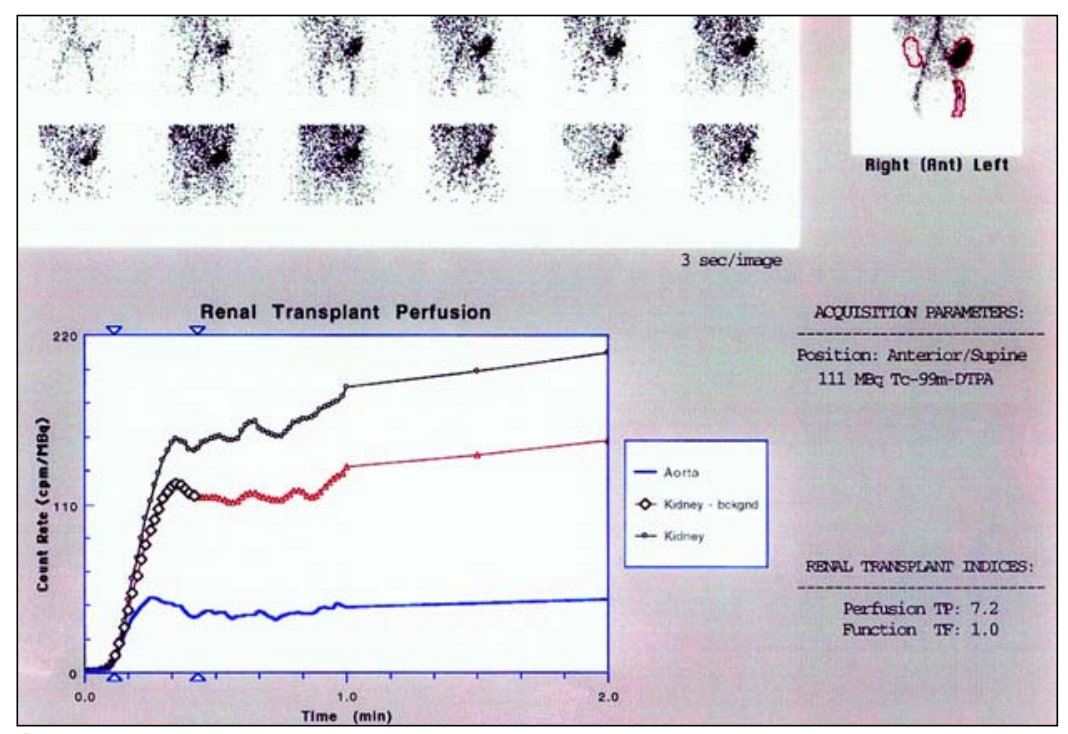

A

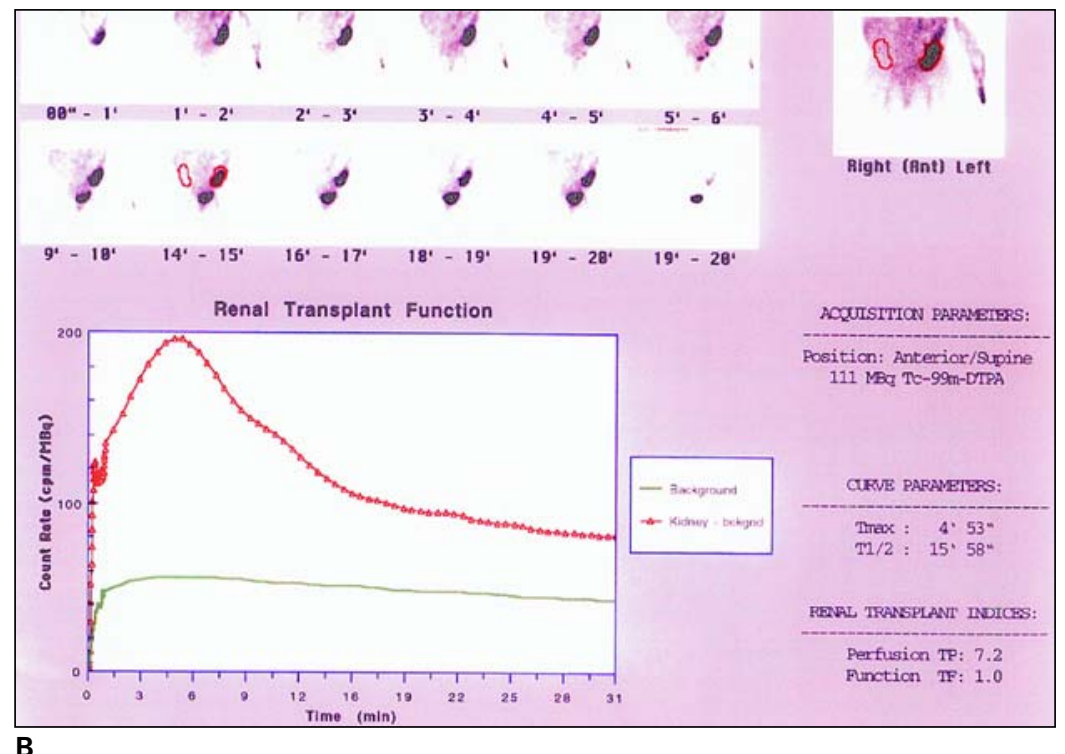

Figura 1. Caso $n^{\circ}$ 6. Cintilografia renal com ${ }^{99 m} T c-D T P A$ mostra padrão normal. A Padrão normal de perfusão imediata do enxerto. B: Padrões normais de filtração e excreção do radiofármaco.

mudanças histopatológicas distintas, assim como quanto ao prognóstico de sobrevida e restabelecimento normal das funções do enxerto ${ }^{(\mathbf{1 2}, 13)}$.

Por convenção, a RA é descrita como a resposta imunológica ao transplante, que normalmente ocorre entre a primeira semana e os três primeiros meses após a cirurgia. Já a RC é responsável pela lenta deterioração da função renal. Os principais fatores envolvidos nos processos de rejeição são os testes de histocom- 


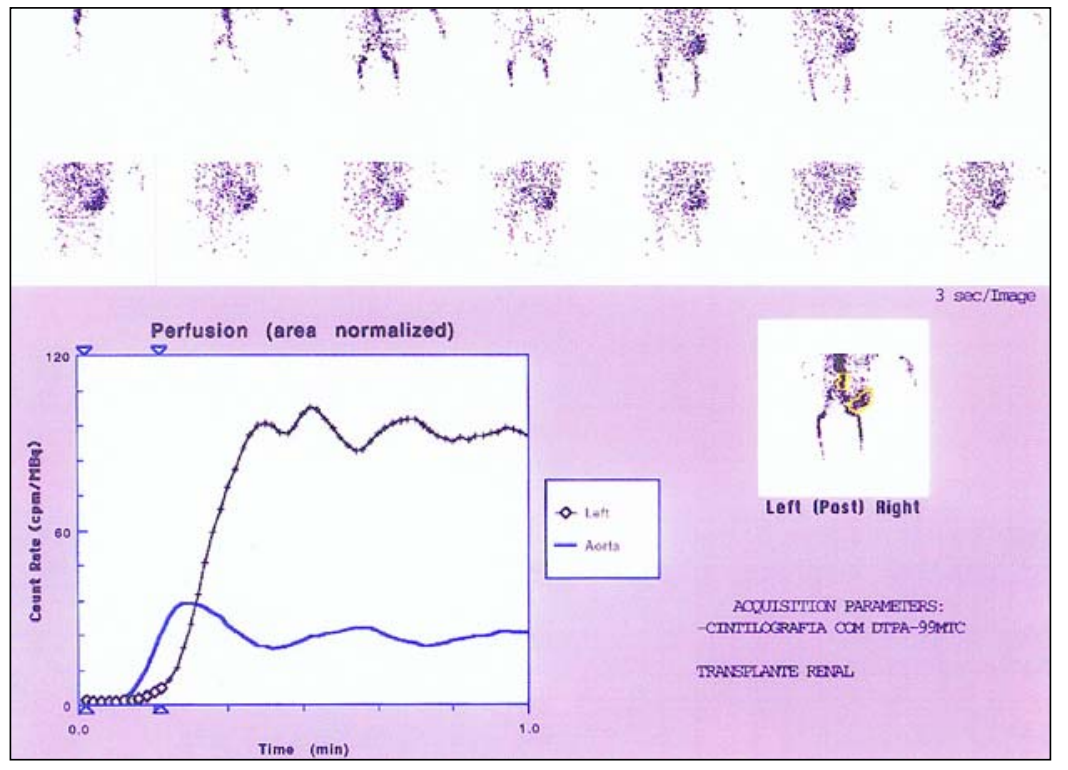

A

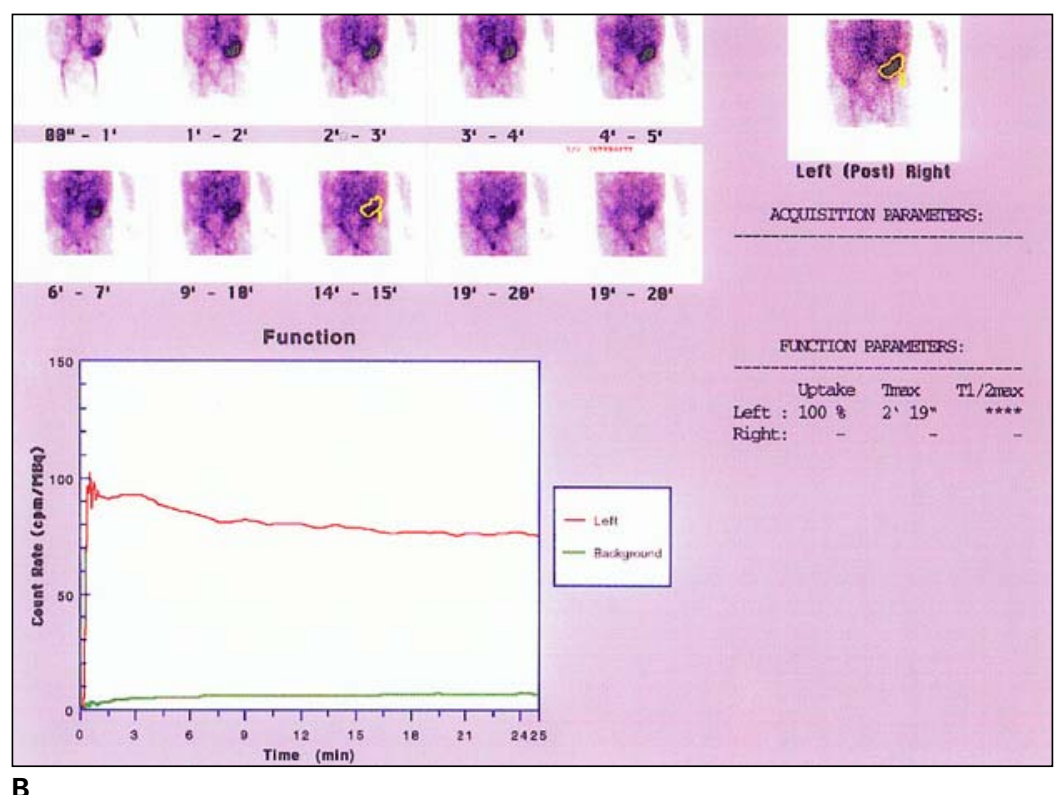

Figura 2. Caso $n^{\circ}$ 52. Cintilografia renal com ${ }^{99 m T C}$ CDTPA mostra padrão de necrose tubular aguda. A Perfusão do enxerto. B: Filtração e excreção do radiofármaco.

patibilidade positivos, a sensibilização do paciente ou o retransplante ${ }^{(\mathbf{1 4})}$.

A literatura aponta para um elevado número de RA, devido aos fatores já citados, além da incompatibilidade imunológica. No entanto, a ocorrência desse quadro vem diminuindo, em conseqüência da utilização dos imunossupressores $^{(\mathbf{1 5})}$. Neste nosso estudo houve predominância da incidência de RA, constituindo 40,4\% das complicações.

Os imunossupressores, administrados sozinhos ou combinados, possibilitam a prevenção da rejeição ao órgão na maioria dos pacientes, sem que para isso causem efeitos colaterais inaceitáveis. Em alguns casos podem ocorrer efeitos colaterais específicos para cada uma das drogas administradas, como, por exemplo, a nefrotoxicidade induzida pela ciclosporina. A prednisolona continua sendo uma das drogas mais prescritas no combate a episódios de rejeição ${ }^{(7)}$. Observamos, em nosso estudo, que $100 \%$ dos pacientes com nefrotoxicidade faziam uso de esquema tríplice de imunossupressão.
Em 1983, desde que foi introduzida na prática clínica, a ciclosporina tornouse a principal droga imunossupressora nos transplantes. Uma série de experimentos demonstrou que esta droga aumentou a sobrevida do enxerto em $20 \%$ durante um ano. Contudo, é uma das drogas mais difíceis de ser manipulada, devido à sua estreita margem terapêutica, variações na absorção e metabolismo de acordo com cada indivíduo, além, é claro, das múltiplas reações adversas que devem ser minuciosamente acompanhadas. A terapia com a ciclosporina é também usada para reduzir a incidência das complicações metabólicas decorrentes do uso de corticóides e minimizar os efeitos colaterais do uso prolongado dos imunossupressores. Dos cinco pacientes com nefrotoxicidade, verificamos três provocados pela ciclosporina, perfazendo $60 \%$ dos pacientes que cursaram com algum tipo de nefrotoxicidade ${ }^{(\mathbf{8 , 1 6 , 1 7 )}}$.

Segundo os parâmetros da medicina nuclear, durante os episódios de rejeição, os estudos cintilográficos renais mostraram que há deterioração da perfusão do enxerto, não sendo observado o radiofármaco no rim, durante os primeiros 60 segundos; nesses casos, a maior parte se encontrava na ilíaca ipsilateral, distal ao transplante. Em imagens posteriores, observou-se que o pouco material radioativo que chegava ao enxerto era eliminado lentamente, e em alguns casos, a quantidade de radiofármaco era tão escassa que a curva nefrográfica representava uma precária função do transplante ${ }^{(9)}$.

Nos exames cintilográficos de pacientes com NTA, durante os primeiros $60 \mathrm{se}$ gundos, a quantidade de material radioativo que atingia o rim era maior do que a que descia pela ilíaca ipsilateral ao enxerto. Na segunda fase do exame observava-se uma filtração satisfatória, embora não se observasse eliminação do radiofármaco. Assim, o nefrograma apresentava a eliminação como uma linha reta, sem a porção excretória da curva ${ }^{(9)}$.

Devido às características do trabalho, o número de cintilografias renais por paciente foi variável, refletindo, entre outros fatores, as complicações suspeitadas e a disponibilidade do serviço para a realização dos exames. 


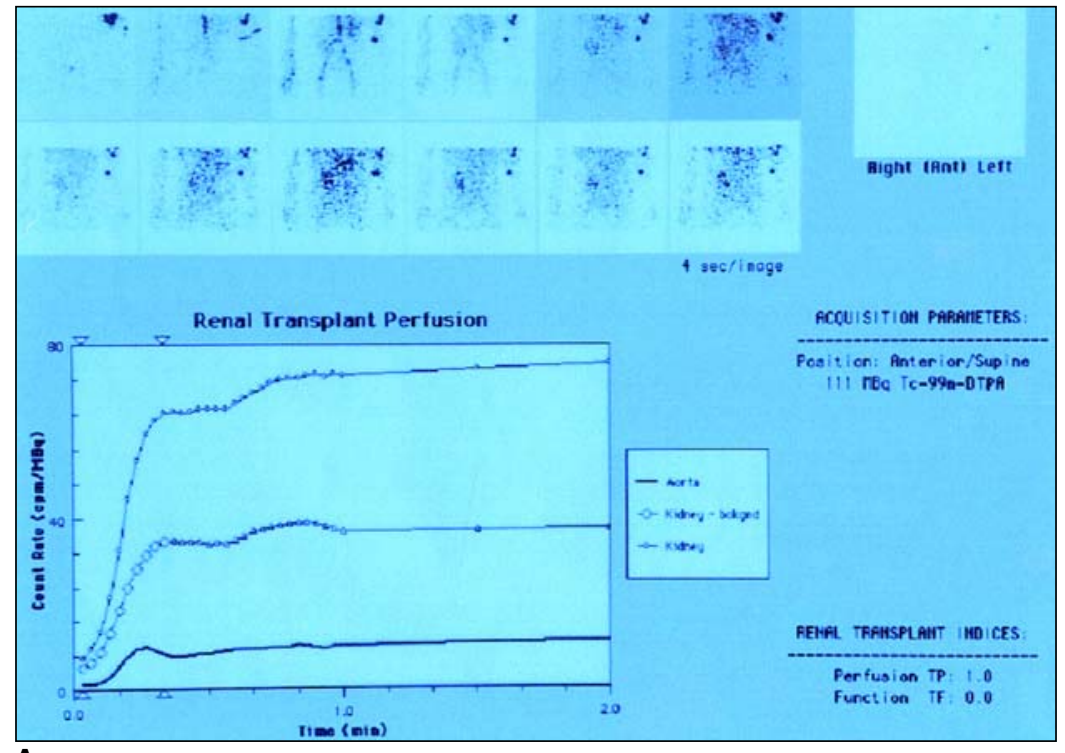

A

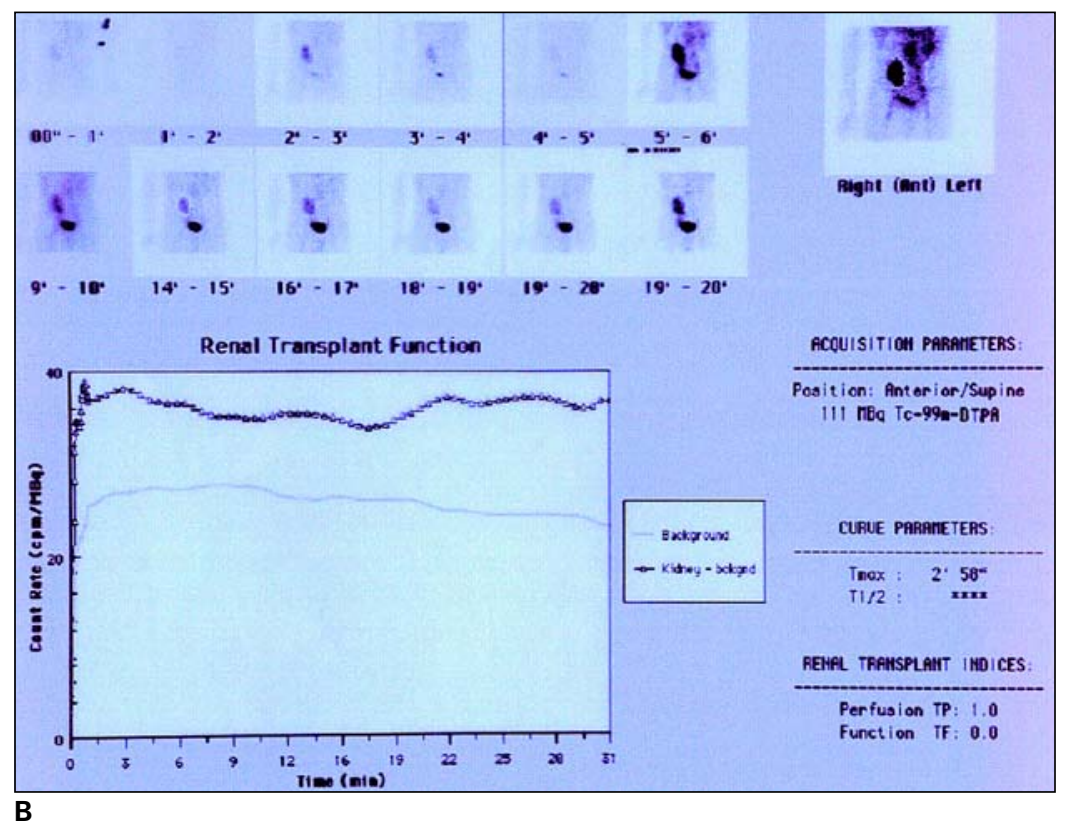

Figura 3. Caso $n^{0}$ 36. Cintilografia renal com ${ }^{99 m} T c-D T P A$ mostra padrões de rejeição. A Fase arterial processando-se em tempo inadequado, alterando a perfusão do enxerto. B: Filtração diminuída e excreção bastante retardada do radiofármaco.

Em nosso estudo tivemos apenas um caso falso-positivo, pela cintilografia renal, que detectou um caso de rejeição ao transplante, não sendo esta confirmada na clínica. Assim, verificamos a fidelidade diagnóstica do exame (sensibilidade de $100 \%$, especificidade de $94,4 \%$, valor preditivo positivo de $97,4 \%$, valor preditivo negativo de $100 \%$ e precisão de imediato, acarretando, assim, um aumento na sobrevida do transplante.

\section{Agradecimentos}

À Faperj, pelo apoio financeiro.

\section{REFERÊNCIAS}

1. Cotran RS, Kumar V, Collins T. Robbins Pathologic basis of disease. 6th ed. Philadelphia: Saunders, 1999.

2. Bejarano MC. Transplante renal: correlação da medicina nuclear com a ultra-sonografia. [Tese de Mestrado]. Rio de Janeiro: Universidade Federal do Rio de Janeiro, 1996.

3. Ellenhorn JDI, Woodle ES, Ghobreal I, Thistlethwaite JR, Bluestone JA. Activation of human T cells in vivo following treatment of transplant recipients with OKT3. Transplantation 1990;50: 608-12.

4. Kreis H, Legendre C, Chatenoud L. OKT3 in organ transplantation. Transplantion Rev 1991;5: 181-99.

5. Mazzucchi E, Lucon AM, Nahas WC, et al. His tological outcome of acute cellular rejection in kidney transplantation after treatment with methylprednisolone. Transplantation 1999;67:430-4.

6. Roitt IM. Essential immunology. 8th ed. London: Blackwell Sci Publ,1998.

7. Allen RDM, Chapman JR. A manual of renal transplantation. London: British Library Cataloguing, 1994.

8. de Ligny BH, Toupance O, Lavaud S, et al. Factors predicting the long-term success of maintenance cyclosporine monotheraphy after kidney transplantation. Transplantation 2000;69:1327-32.

9. Fonseca LM. Uma contribuição à avaliação da função renal. [Tese de Doutorado]. Rio de Janeiro: Universidade Federal do Rio de Janeiro, 1990.

10. Fonseca LMB, Martins FPP, Cadorna MAC, Gutfilen B. Nuclear medicine functional evaluation of renal transplantation. Nucl Med Comm 1999; 20:962.

11. Kon SP, Templar J, Dodd SM, Rudge CJ, Raftery MJ. Diagnostic contribution of renal allograft biopsies at various intervals after transplantation. Transplantation 1997;63:547-50.

12. Marcén R, Orofino L, Pascual J, et al. Delayed graft function does not reduce the survival of renal transplant allografts. Transplantation 1998;66:461-6.

13. O'Reilly PH, Shields RA, Testa HJ. Nuclear medicine in urology and nephrology. London: Butterworths, 1991.

14. Gaber LW, Moore LW, Gaber AO, et al. Utility of standardized histological classification in the management of acute rejection. 1995 Efficacy Endpoints Conference. Transplantation 1998; 65:37680.

15. Macphee IAM, Bradley JA, Briggs JD, et al. Longterm outcome of a prospective randomized trial of convertion from cyclosporine to azathioprine treatment one year after renal transplantation. Transplantation 1998;66:1186-92.

16. Ferreira U, Lima ML, Fucsietal M. Transplante renal - aspectos atuais. Rev Bras Med 1993;50: 1499-507.

17. Solomon H, Gonwa TA, Mor E, et al. OKT3 rescue for steroid-resistant rejection in adult liver transplantation. Transplantation 1993;55:87-91. 